\title{
Kondo Resonance in the Presence of Spin-Polarized Currents
}

\author{
Yunong Qi, ${ }^{1}$ Jian-Xin Zhu, ${ }^{2, \text { * }}$ Shufeng Zhang, ${ }^{3}$ and C. S. Ting, ${ }^{1}$ 丹 \\ ${ }^{1}$ Texas Center for Superconductivity, University of Houston, Houston, Texas 77204 \\ ${ }^{2}$ Theoretical Division, Los Alamos National Laboratory, Los Alamos, New Mexico 87545 \\ ${ }^{3}$ Department of Physics \& Astronomy, University of Missouri, Columbia, Missouri 65211
}

(Dated: October 24, 2018)

\begin{abstract}
We propose an improved method of the equation of motion approach to study the Kondo problem in spin-dependent non-equilibrium conditions. We find that the previously introduced additional renormalization for non-equilibrium Kondo effects is not required when we use a proper decoupling scheme. Our improved formulation is then applied to address the spin-split Kondo peaks when a spin current injects into a Kondo system.
\end{abstract}

PACS numbers: 75.20.Hr, 72.15.Qm, 72.25.-b, 85.75.-d

The Kondo effect [1] has been one of the central and challenging problems in condensed matter physics for many years. In equilibrium, the Kondo peak formed at the Fermi level is degenerate with respect to spin degrees of freedom when a magnetic impurity is embedded in a non-magnetic metal. By applying a magnetic field, the spin degeneracy of the Kondo peak can be lifted due to Zeeman splitting of the impurity level. For a mesoscopic system such as a quantum dot, one can also use ferromagnetic leads to lift the spin degeneracy; this is because the ferromagnetic leads provide the spin dependence of the interaction (or hybridization) between the conduction electrons of the leads and the localized state in the quantum dot. To explain the above spin-dependent non-equilibrium phenomena, one frequently relies on the method of the equation motion (EOM) where an additional renormalization in the self-energy is introduced without rigorous justification.

In this Letter, we address two issues. First, we show that the non-equilibrium Kondo problem can be solved without the artificial additional renormalization as long as one correctly keeps the previously neglected terms in the EOM. We establish a new formulation for the case of finite and infinite $U$ in the Anderson model. Second, we use the improved EOM method to predict spin-split Kondo peaks in the presence of a non-equilibrium spin accumulation. The spin accumulation has played a crucial role in the emerging field of spin electronics [2, 3,, 4 ]. Our prediction directly connects the separation of the Kondo peaks with the spin accumulation, and thus it provides a way to determine the spin accumulation in Kondo systems. We emphasize an important distinction of the present study from previous one: the non-equilibrium condition generated by a voltage across the quantum dot and the ferromagnetic lead has a common chemical potential for spin up and down electrons, while the spin accumulation generates spin-split chemical potentials.

There are a number of methods to study the Kondo effect in the spin-dependent non-equilibrium condition. The EOM approach of the Anderson model has been used for treating both equilibrium and non-equilibrium Kondo physics at low temperatures. The EOM approach includes re-summation of low-order hopping processes and needs a decoupling scheme in order to obtain a closed analytical form. We follow the procedure introduced by Appelbaum, Penn, and Lacroix (APL) [5, 6], which is known to capture the right qualitative feature of physics at low temperatures. We note that other approaches, e.g., numerical renormalization group method, can also describe the low temperature Kondo effect [7, 8].

The Hamiltonian of the impurity Anderson model is

$$
\begin{aligned}
H=\sum_{k, \sigma} \epsilon_{k \sigma} c_{k \sigma}^{\dagger} c_{k \sigma} & +\sum_{\sigma} \epsilon_{d \sigma} d_{\sigma}^{\dagger} d_{\sigma}+U d_{\sigma}^{\dagger} d_{\sigma} d_{\bar{\sigma}}^{\dagger} d_{\bar{\sigma}} \\
& +\sum_{k, \sigma} V_{k \sigma}\left[c_{k \sigma}^{\dagger} d_{\sigma}+d_{\sigma}^{\dagger} c_{k \sigma}\right] .
\end{aligned}
$$

Here $c_{k \sigma}^{\dagger}$ and $d_{\sigma}^{\dagger}$ are respectively the creation operators for conduction and $d$ electrons at the impurity site. The quantities $\epsilon_{k \sigma}, \epsilon_{d \sigma}$ are the conduction electron energy dispersion and the impurity energy, respectively. We assume that conduction electrons density of states is constant but spin dependent, i.e., $\rho\left(\epsilon_{\sigma}\right)=1 / 2 D_{\sigma}$ when $-D_{\sigma} \leq \epsilon_{k \sigma} \leq D_{\sigma}, U$ is the intra-atomic Coulomb interaction at the impurity site, and $V_{k \sigma}$ represents the $s$ - $d$ hybridization.

By using the standard procedure for the EOM, we obtain the impurity Green function, $G_{d \sigma} \stackrel{\text { def }}{=} \ll d_{\sigma} \mid d_{\sigma}^{\dagger} \gg$,

$$
\begin{gathered}
G_{d \sigma}=\frac{1-\bar{n}_{d \bar{\sigma}}(\omega)}{\omega-\epsilon_{d \sigma}-\Sigma_{0 \sigma}+\frac{U \Sigma_{1 \sigma}}{\omega-\epsilon_{d \sigma}-U-\Sigma_{0 \sigma}-\Sigma_{3 \sigma}}} \\
+\frac{\bar{n}_{d \bar{\sigma}}}{\omega-\epsilon_{d \sigma}-\Sigma_{0 \sigma}-U-\frac{U\left[\Sigma_{3 \sigma}-\Sigma_{1 \sigma}\right]}{\omega-\epsilon_{d \sigma}-U-\Sigma_{0 \sigma}-\Sigma_{3 \sigma}}}
\end{gathered}
$$

To arrive at Eq. (2), we have made the decoupling approximation procedure shown below. The "dynamic" average occupation number (i.e., frequency-dependence) of the impurity level is defined as

$$
\bar{n}_{d \bar{\sigma}}(\omega) \stackrel{\text { def }}{=}\left\langle n_{d \bar{\sigma}}\right\rangle-\sum_{q} \frac{V_{q \bar{\sigma}}\left\langle c_{q \bar{\sigma}}^{\dagger} d_{\bar{\sigma}}\right\rangle}{D_{1 \sigma}(\omega, q)}+\sum_{q} \frac{V_{q \bar{\sigma}}^{*}\left\langle d_{\bar{\sigma}}^{\dagger} c_{q \bar{\sigma}}\right\rangle}{D_{2 \sigma}(\omega, q)},
$$


and the three self-energies are

$$
\begin{gathered}
\Sigma_{0 \sigma} \stackrel{\text { def }}{=} \sum_{k} \frac{\left|V_{k \sigma}\right|^{2}}{\omega-\epsilon_{k \sigma}}, \\
\Sigma_{1 \sigma} \stackrel{\text { def }}{=} \sum_{k} \sum_{q}\left[\frac{V_{k \bar{\sigma}}^{*} V_{q \bar{\sigma}}\left\langle c_{q \bar{\sigma}}^{\dagger} c_{k \bar{\sigma}}\right\rangle}{D_{2 \sigma}(\omega, k)}+\frac{V_{k \bar{\sigma}} V_{q \bar{\sigma}}^{*}\left\langle c_{k \bar{\sigma}}^{\dagger} c_{q \bar{\sigma}}\right\rangle}{D_{1 \sigma}(\omega, k)}\right] \\
+\sum_{k}\left[\frac{V_{k \bar{\sigma}}^{*}\left\langle c_{k \bar{\sigma}} d_{\bar{\sigma}}^{\dagger}\right\rangle}{D_{2 \sigma}(\omega, k)}+\frac{V_{k \bar{\sigma}}\left\langle c_{k \bar{\sigma}}^{\dagger} d_{\bar{\sigma}}\right\rangle}{D_{1 \sigma}(\omega, k)}\right] \Sigma_{0 \sigma},
\end{gathered}
$$

and

$$
\Sigma_{3 \sigma} \stackrel{\text { def }}{=} \sum_{k} \frac{\left|V_{k \bar{\sigma}}\right|^{2}}{D_{1 \sigma}(\omega, k)}+\sum_{k} \frac{\left|V_{k \bar{\sigma}}\right|^{2}}{D_{2 \sigma}(\omega, k)},
$$

where

$$
D_{1 \sigma}(\omega, k) \stackrel{\text { def }}{=} \omega+\epsilon_{k \bar{\sigma}}-\epsilon_{d \sigma}-\epsilon_{d \bar{\sigma}}-U
$$

and

$$
D_{2 \sigma}(\omega, k) \stackrel{\text { def }}{=} \omega-\epsilon_{k \bar{\sigma}}-\epsilon_{d \sigma}+\epsilon_{d \bar{\sigma}} .
$$

We shall point out that the expectation value of $\left\langle c_{q \bar{\sigma}}^{\dagger} c_{k \bar{\sigma}}\right\rangle$, $\left\langle d_{\bar{\sigma}}^{\dagger} c_{k \bar{\sigma}}\right\rangle$ which have been discarded in previous EOM studies are important at low temperatures since they diverge logarithmically at the Fermi level as the temperature approaches to zero. Their values should be selfconsistently evaluated through the following identities:

$$
\left\langle c_{q \bar{\sigma}}^{\dagger} c_{k \bar{\sigma}}\right\rangle=-\frac{1}{\pi} \int f_{F D}(\omega) \operatorname{Im} \ll c_{k \bar{\sigma}} \mid c_{q \bar{\sigma}}^{\dagger} \gg d \omega,
$$

where $f_{F D}(\omega)=1 /\left[e^{\beta \omega}+1\right]$ is the Fermi-Dirac distribution function, $\beta=1 / k_{B} T$, and the Green function $\ll c_{k \bar{\sigma}} \mid c_{q \bar{\sigma}}^{\dagger} \gg$ is:

$$
\ll c_{k \bar{\sigma}} \mid c_{q \bar{\sigma}}^{\dagger} \gg=\frac{\delta_{q, k}}{\omega-\epsilon_{k \bar{\sigma}}}+\frac{V_{k \bar{\sigma}} V_{q \bar{\sigma}}^{*} \ll d_{\bar{\sigma}} \mid d_{\bar{\sigma}}^{\dagger} \gg}{\left(\omega-\epsilon_{k \bar{\sigma}}\right)\left(\omega-\epsilon_{q \bar{\sigma}}\right)},
$$

and similarly

$$
\left\langle d_{\bar{\sigma}}^{\dagger} c_{k \bar{\sigma}}\right\rangle=-\frac{1}{\pi} \int f(\omega) \operatorname{Im} \ll c_{k \bar{\sigma}} \mid d_{\bar{\sigma}}^{\dagger} \gg d \omega,
$$

with

$$
\ll c_{k \bar{\sigma}} \mid d_{\bar{\sigma}}^{\dagger} \gg=\frac{V_{k \bar{\sigma}} \ll d_{\bar{\sigma}} \mid d_{\bar{\sigma}}^{\dagger} \gg}{\left(\omega-\epsilon_{k \bar{\sigma}}\right)} .
$$

Equation (2) is an extension to a similar result obtained by Meir et al. [9]. Equations (2) through (10) constitute a set of the closed self-consistent equations which can be numerically solved. Before we carry out numerical calculations, we point out the new ingredients in our

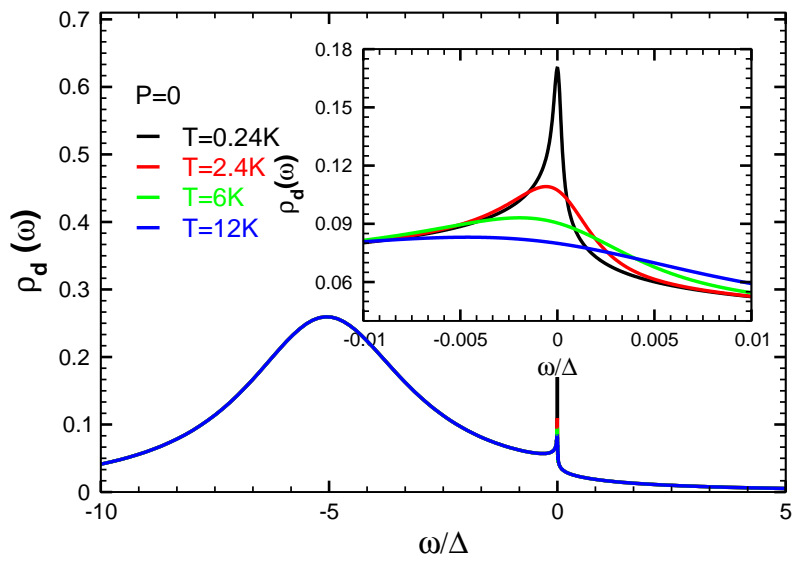

Figure 1: (Color) Spectral density $\rho_{d \sigma}$ calculated via the EOM method for an infinity $U$ Anderson model for various temperatures in the absence of spin polarization. The inset displays the zoom-in view of the Kondo resonance near the Fermi energy.
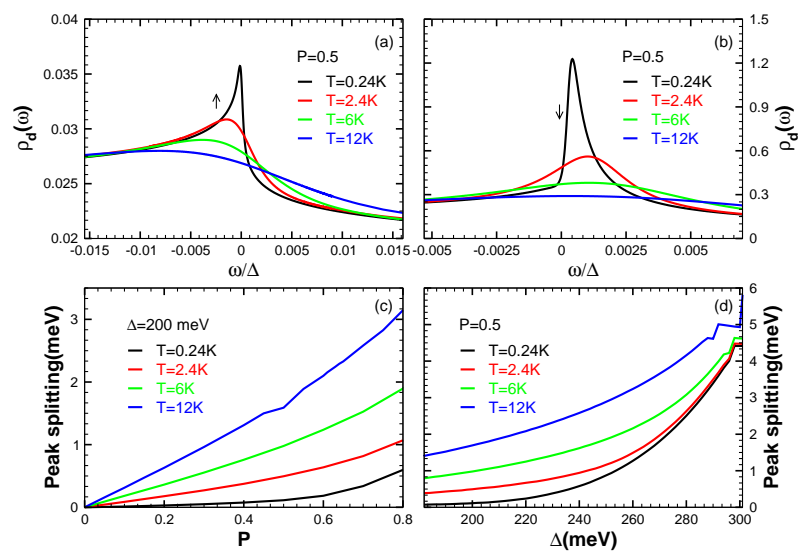

Figure 2: (Color) Spectral density $\rho_{d \sigma}$ calculated via the EOM method for an infinity $U$ Anderson model for various temperatures for a fixed degree, $P=0.5$, of spin polarization (a) and (b), and the Kondo resonance splitting as a function of the degree of spin polarization (c) and the hybridization strength at a fixed $P=0.5(\mathrm{~d})$.

formula: 1) the effective occupancy is frequency dependent, and 2) the higher order self-energy contains the intermediate off-diagonal states in momentum space (e.g., $\left.\left\langle c_{k \sigma}^{\dagger} c_{q \sigma}\right\rangle\right)$ and charge fluctuations (e.g., $\left.\left\langle d_{\sigma}^{\dagger} c_{q \sigma}\right\rangle\right)$. Solving the coupled Eq. (2)-(10) not only yields the Kondo resonance at low temperatures but also allows us to explicitly include the logarithmic divergence in general.

For the case of an infinite $U$, the Eq. (2) takes a simpler 
form

$$
G_{d \sigma}=\frac{1-\left\langle n_{d \bar{\sigma}}(\omega)\right\rangle+\sum_{q} \frac{V_{q \bar{\sigma}}\left\langle d_{\bar{\sigma}}^{\dagger} c_{q \bar{\sigma}}\right\rangle}{D_{2 \sigma}(\omega, q)}}{\omega-\epsilon_{d \sigma}-\alpha \Sigma_{0 \sigma}-\sum_{k} \sum_{q} \frac{V_{k \bar{\sigma}}^{*} V_{q \bar{\sigma}}\left\langle c_{q \bar{\sigma}}^{\dagger} c_{k \bar{\sigma}}\right\rangle}{D_{2 \sigma}(\omega, k)}},
$$

where the zero-ordered self-energy is renormalized by a factor

$$
\alpha=1+\sum_{k} \frac{V_{k \bar{\sigma}}^{*}\left\langle c_{k \bar{\sigma}} d_{\bar{\sigma}}^{\dagger}\right\rangle}{D_{2 \sigma}(\omega, k)} .
$$

Before we apply our formulation to discuss the Kondo resonance in the presence of the spin accumulation, we examine various well-studied cases by numerically solving Eq. (13). We choose the following parameters for our numerical calculation. The energy of the half-width of the impurity resonance in a nonmagnetic metal, $\Delta_{0}=$ $-\operatorname{Im}\left[\Sigma_{0 \sigma}\left(\omega+i 0^{+}\right)\right]$, is taken $200 \mathrm{meV}$ unless specified otherwise. The conduction band half-width is $D=100 \Delta_{0}$. The degenerate impurity level is $\epsilon_{d}=-6 \Delta_{0}$.

The simplest case is the conventional equilibrium Kondo problem where neither impurity state nor hybridization is spin-dependence. Figure 1 shows the impurity spectral density $\left(\rho_{d \uparrow}=\rho_{d \downarrow}\right)$ for four temperatures. As expected, the virtual bound level with a broad spectrum and a sharp peak at the Fermi level known as the Kondo peak appears. The Kondo peak is suppressed and broadened when the temperature is increased. The Kondo temperature which is defined as the full-width of the Kondo peak is $T_{K} \simeq \exp \left[\pi \epsilon_{d} / 2 \Delta_{0}\right]$. These wellknown results agree with many various approaches, e.g., the scaling analysis 11 and the non-crossing approximation [12]. Meir et al. [10] and Martinek et al. [13] has also used the EOM approach to derive these Kondo peaks in the absence of spin polarization.

We now turn on the spin polarization. In a quantum dot, the spin polarization is introduced via the coupling to a ferromagnetic lead. In this case, one can parametrize the spin dependence through the hybridization parameter, i.e., $\Delta_{\sigma}=\Delta_{0}(1-\sigma P)$, where the parameter $0<P<1$ and $\sigma= \pm 1$ for spin up $(+1)$ and down $(-1)$. The results are shown in Fig. 2, When $P$ is non-zero, the Kondo resonance splits [Fig. 2(a)-(b)], i.e., the peak for spin up (down) spectral density shifts below (above) the Fermi energy. Notice also that the intensity of the peak for spin up (down) is suppressed (enhanced) compared to the equilibrium Kondo peaks, see Fig. 2(a)(b) and Fig. 1. These results are again consistent with those obtained by Martinek et al. [13]. Another way of introducing the spin-polarization is to apply a magnetic field. In this case, the Zeeman splitting of the impurity level becomes $\epsilon_{\sigma}=\epsilon_{d}+\sigma \mu_{B} B$, where $B$ is the magnetic field. We find that the Kondo peaks for spin up and down are separated by $2 \mu_{B} B$ (not shown), which agrees with that in Ref. 10 .

The agreement of our results with other approaches validates the EOM approach of Eq. (2) or Eq. (13). The significance of our EOM approach is that it does not rely on the additional renormalization introduced in the previous EOM technique [9]. The purpose of the additional renormalization is to account for the spin dependent level splitting and broadening [10, 13]. The lack of rigorous justification for the existence of the additional renormalization has cast a doubt for the effectiveness of the EOM approach for the non-equilibrium Kondo problem. In our improved EOM formula, we have shown that the correct Kondo resonance can be derived without introducing the additional renormalization. Comparing with previous calculations, we have properly evaluated terms such as $\left\langle c_{q \bar{\sigma}}^{\dagger} c_{k \bar{\sigma}}\right\rangle,\left\langle d_{\bar{\sigma}}^{\dagger} c_{k \bar{\sigma}}\right\rangle$ through Eq. (5). These terms make crucial contributions to the Kondo resonance splitting at low temperatures. Neglecting these terms will lead to severe errors, which has to be recovered by the artificially adding an additional renormalization.

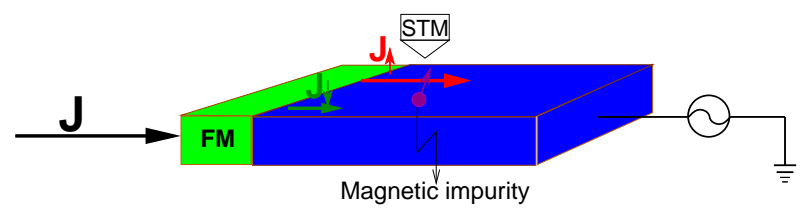

Figure 3: (Color) Schematic illustration of a spin-current injected from a ferromagnetic layer to a non-magnetic conductor containing a magnetic impurity.

We now apply the EOM equation to calculate the Kondo resonance in the presence of the spin accumulation. We consider a bilayer structure where a current flows from the ferromagnetic layer to the nonmagnetic layer containing Kondo impurities as schematically shown in Fig. 3. When a spin-polarized current injects into the non-magnetic conductor, a spin accumulation is built-up near the interface. Assuming that the ferromagnetic layer carries a spin-polarization of the current $P$, the spin accumulation is [14, 15] $\delta m=$ $\left(P j \lambda \mu_{B} / e D\right) \exp (-x / \lambda)$ where $j$ is the current density, $\lambda$ is the spin diffusion length, $\mu_{B}$ is Bohr magneton, $D$ is the diffusion constant, and $x$ is the distance away from the interface. If we only consider the Kondo impurity sufficiently close to the interface, i.e., within the distance of $\lambda$, we can drop the spatial dependence of the spin accumulation, i.e., $\delta m=P j \lambda \mu_{B} / e D$. To calculate the Kondo resonance from Eq. (13), we specify the dependence of the parameters on the spin accumulation. First, the spin accumulation makes the chemical potential spin-dependent [14, 15]. Specifically, the chemical potential splitting of spin up and down conduction electron is $\mu^{\uparrow}-\mu^{\downarrow}=\delta m\left(e D \rho / \mu_{B}\right)=\operatorname{Pj} \rho \lambda$ [16], where $\rho$ is the resistivity. Thus one should replace the Fermi 


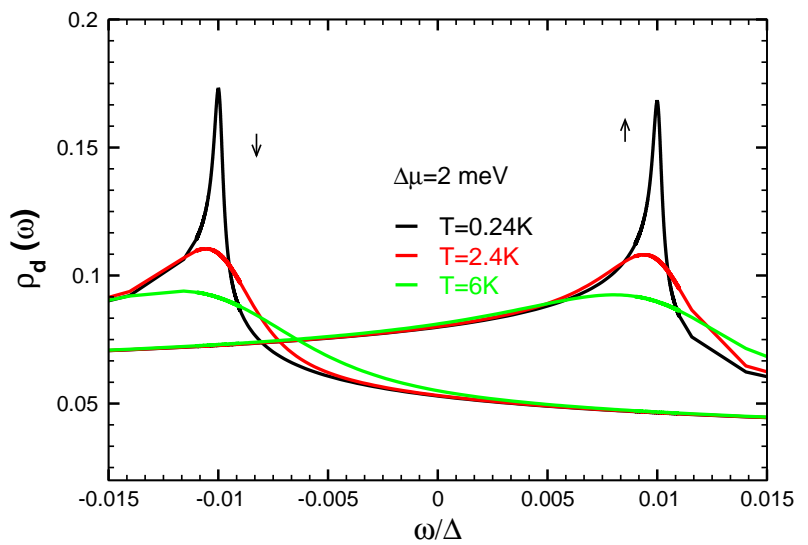

Figure 4: (Color) Spectral density $\rho_{d \sigma}$ calculated via the EOM method for an infinity $U$ Anderson impurity for various temperatures in presence of spin accumulation with $\Delta \mu=2 \mathrm{meV}$.

level in Eq. (13) by the spin-dependent chemical potentials for the spin up and down $\mu^{\uparrow}=E_{F}+P j \rho \lambda / 2$ and $\mu^{\downarrow}=E_{F}-\operatorname{Pj} \rho \lambda / 2$, where $E_{F}$ is the Fermi level. Second, the hybridization parameter $\Delta$ would also be spin dependent since the density of states of the conduction electrons is modified by the non-equilibrium electrons. However, the non-equilibrium electron density at very high current density (say $10^{7} \mathrm{~A} / \mathrm{cm}^{2}$ ) is at least several orders of magnitude smaller than the equilibrium electron density, and thus the correction to $\Delta$ is very small and we will assume that $\Delta$ remains spin-independent. Finally, the spin accumulation could lead to the spindependent energy shift of the impurity state. If one models the interaction between the spin accumulation and the impurity via a phenomenological exchange coupling, i.e., $H^{\prime}=-J_{e x} \delta \mathbf{m} \cdot \mathbf{S}_{i}$, where $\delta \mathbf{m}$ is the spin accumulation and $\mathbf{S}_{i}$ is the impurity spin, the impurity level would be spin-split; this will be equivalent to the case when the impurity is subject to an effective magnetic field $\mathbf{B}_{e f f}=J_{e x} \delta \mathbf{m}$. Although the magnitude of the effective field could be respectable for a high current density, the local level splitting by a magnetic field has already been thoroughly investigated and thus we neglect the effect of the direct coupling between the spin accumulation and the impurity. Therefore, we focus on the Kondo resonances due to spin dependent Fermi levels. In Fig. 团 we show the spectral density $\rho_{d \sigma}$ for various temperatures with a spin-current-induced chemical potential shift. It is found that the spin-current influence on the Kondo resonance splitting is robust against temperatures. The more the amplitude of chemical potential relative shift by spin current, the more pronounced the Kondo resonance splitting between two spin channels will be. Noticeably, the amplitude of the split Kondo peaks remains robust against the spin-induced chemical potential splitting (compare Fig. (4 with Fig. 1), which is different from the case of a ferromagnetic metal as discussed before (see
Fig. 2(a)-(b)). This observation is the hallmark of the Kondo resonance splitting from the spin accumulation - a purely non-equilibrium effect.

Advanced experimental techniques such as magnetic tunneling into quantum dots and STM measurement would be able to detect the spin-split Kondo peaks. This will provide a high resolution detection of the spin accumulation. For example, for a relatively small current density of the order of $10^{5} \mathrm{~A} / \mathrm{cm}^{2}$, the Kondo peaks will be separated by about $\delta E=0.02 \mathrm{meV}$ for a typical transition metal such as Fe (note that the width of the Kondo resonance, which is of the order of the Kondo temperature). In comparison, the reliable measurement of the spin accumulation based on the Silsbee-Johnson technique [14] usually requires the current density more than $10^{7} \mathrm{~A} / \mathrm{cm}^{2}$ in order to derive sufficient large signals.

In conclusion, we have developed an improved decoupling scheme for the EOM approach in Kondo problems at low temperature. We show that by keeping the previously discarded terms in the EOM approach, the additional renormalization is naturally implemented. We then apply the improved EOM approach to study the Kondo effect in the presence of the spin accumulation. We predict that the Kondo peaks are spin-split due to the spin dependence of the chemical potentials.

We are grateful to M. E. Flatté and Li Sheng for helpful discussions and suggestions. This work was supported by the Robert Welch Foundation No. E-1146 at the University of Houston (Y.Q. and C.S.T.), by U.S. DOE under Contract No. DE-AC52-06NA25396 and under Grant Nos. LDRD-DR X9GT \& X9HH (J.X.Z.), and by NSFDMR-074182 (S.Z.) at the University of Missouri.

* Electronic address: jxzhu@lanl.gov URL: http://theory.lanl.gov

† Electronic address: csting@mail.uh.edu

[1] A. C. Hewson, The Kondo Problem to Heavy Fermions (Cambridge University Press, Cambridge, U.K., 1993).

[2] S. Wolf et. al., Science 294, 1488 (2001).

[3] R. Fiederling et. al., Nature (London) 402, 787(1999).

[4] B. E. Kane, Nature(London) 393, 133 (1998).

[5] J. A. Appelbaum and D. R. Penn, Phys. Rev. 188, 874 (1969).

[6] C. Lacroix, J. Phys. F: Metal Phys. 11, 2389 (1981).

[7] J. Martinek et al., Phys. Rev. Lett. 91, 247202 (2003).

[8] M.-S. Choi, D. Sanchez, and R. Lopez, Phys. Rev. Lett. 92, 056601 (2004).

[9] Y. Meir, N. S. Wingreen, and P. A. Lee, Phys. Rev. Lett. 66, 3048 (1991).

[10] Y. Meir, N. S. Wingreen, P. A. Lee, Phys. Rev. Lett. 70, 2601 (1993).

[11] F. D. M. Haldane, Phys. Rev. Lett. 40, 416 (1978).

[12] N. E. Bickers, Rev. Mod. Phys. 59, 845 (1987).

[13] J. Martinek et al., Phys. Rev. Lett. 91, 127203 (2003).

[14] M. Johnson and R. H. Silsbee, Phys. Rev. Lett. 55, 1790 (1985); M. Johnson, Phys. Rev. Lett. 70, 2142 (1993); 
[15] T. Valet and A. Fert, Phys. Rev. B 48, 7099 (1993).

Columbia, 2003.

[16] Yunong Qi, Ph.D. Thesis University of Missouri at 